\title{
Projective manifolds dominated by abelian varieties
}

\section{Jun-Muk Hwang ${ }^{1}$ and Ngaiming Mok ${ }^{2}$}

In [La], Lazarsfeld showed that if there exists a surjective morphism from the projective space $\mathbf{P}_{n}$ to a projective manifold $X$, then $X \cong \mathbf{P}_{n}$. A natural generalization of this would be the study of projective manifolds which can be the target of a surjective morphism from homogeneous spaces.

In [HM2], the authors studied the question for a rational homogeneous space $S$ of Picard number 1. From the condition on the Picard number, a non-constant morphism $f: S \rightarrow X$ must be a finite morphism. The main result of [HM2] says that if $f: S \rightarrow X$ is a finite morphism with nonempty ramification onto a projective manifold, then $X \cong \mathbf{P}_{n}$.

Debarre studied the question for a simple abelian variety $A$ ([De]). Since $A$ is simple, a non-constant morphism $f: A \rightarrow X$ must be a finite morphism. His main result says that if $f: A \rightarrow X$ is a finite morphism with nonempty ramification from a simple abelian variety onto a projective manifold, then $X \cong \mathbf{P}_{n}$. He used the fact that curves in a simple abelian variety have ample normal bundles, making it possible to apply Mori's characterization of $\mathbf{P}_{n}([\mathrm{Mo}])$ as in the argument in Lazarsfeld's proof for $\mathbf{P}_{n} \rightarrow X$.

In this article, we study the same question for an arbitrary abelian variety $A$. Note that if $f: A \rightarrow B$ is a surjective morphism with connected fibers onto a normal variety $B$, then vector fields of $A$ descend to $B$ as derivations of $f_{*} \mathcal{O}_{A}=\mathcal{O}_{B}$ and $B$ must be a quotient abelian variety. By Stein factorization, any surjective morphism $f: A \rightarrow X$ to a projective manifold can be factored through a finite morphism on some quotient abelian variety of $A$. So to study target manifolds $X$ we may restrict to the case of finite morphisms. For $X$ of Picard number 1 we prove

Theorem 1 Let $f: A \rightarrow X$ be a finite morphism with nonempty ramification from an abelian variety onto a projective manifold $X$ of Picard number 1 . Then, $X \cong \mathbf{P}_{n}$.

When $X$ is not assumed to be of Picard number 1, and $A$ is not simple, then $X$ may be more complicated than $\mathbf{P}_{n}$, e.g., $X$ can be a bundle of projective spaces over some abelian variety. Our Main Theorem says that these are essentially the only possibilities.

Main Theorem Let $f: A \rightarrow X$ be a finite morphism with nonempty ramification from an abelian variety onto a projective manifold $X$. Then $X$ is a holomorphic bundle of projective spaces over a projective algebraic manifold $Y$ of smaller dimension and there exists a finite morphism

\footnotetext{
${ }^{1}$ Supported by Grant No. 98-0701-01-5-L from the KOSEF.

${ }^{2}$ Supported by an RGC-grant of the Hong Kong Research Grant Council
} 
from an abelian variety onto $Y$. Thus there exist a finite sequence of surjective morphisms between projective manifolds $\psi_{i}: X_{i-1} \rightarrow X_{i}, 1 \leq i \leq N$ with $X_{0}=X$ such that each $\psi_{i}$ is a holomorphic $\mathbf{P}_{k_{i}}$-bundle for some integer $k_{i}, 1 \leq i \leq N$ and $X_{N}$ has a finite unramified cover by an abelian variety.

As is well-knwon, $X_{N}$ in the above statement need not be an abelian variety (e.g. [Ue, p.208]).

As opposed to the case of simple abelian varieties, the fundamental difficulty of our problem arises from the fact that the normal sheaf of an algebraic curve on $A$ need not be ample. (This difficulty arises even if $X$ is of Picard number 1.) Deformation of minimal rational curves on $X$ then leads to multi-valued distributions. We combine deformation theory of rational curves on $X$ with topological considerations to handle multi-valuedness of such distributions. In addition to providing the key step to our solution of the Main Theorem, our treatment of multi-valued distributions furnishes an ingredient pertinent to the general theory of distributions defined by minimal rational curves, a systematic study of which just started in [HM1].

\section{Varieties of minimal rational tangents on projective manifolds dominated by abelian varieties}

In this section, we will prove some basic results about the deformation theory of rational curves on projective manifolds dominated by abelian varieties. To start with,

Proposition 1 Let $f: A \rightarrow X$ be a finite morphism with nonempty ramification from an abelian variety onto a projective manifold $X$. Then $X$ is uniruled.

Proof. By the adjunction formula, $K_{A}=f^{*} K_{X}+R$ where $R$ is the ramification divisor. By assumption, $R \neq \emptyset$. Choose a generic curve $C \subset A$ which meets $R$. Then $f(C)$ is a curve through a generic point on $X$, with $K_{X} \cdot f(C)<0$. By [MM], this implies that $X$ is uniruled.

Let $X$ be a uniruled projective manifold. Among components of the Hilbert scheme of rational curves on $X$ whose members cover $X$, we choose one component $\mathcal{K}$ of minimal degree. $\mathcal{K}$ will be called a minimal rational component. A member of $\mathcal{K}$ will be called a $\mathcal{K}$-curve. Let $\mathcal{K}_{x} \subset \mathcal{K}$ be the subscheme consisting of $\mathcal{K}$-curves passing through a generic point $x \in X$. By the minimality of the degree, $\mathcal{K}_{x}$ is complete. Generic members of $\mathcal{K}_{x}$ are smooth at $x$, defining a rational map $\Phi_{x}: \mathcal{K}_{x} \rightarrow \mathbf{P} T_{x}(X)$ by associating the tangent vector at $x$ to each curve smooth at $x$. The strict image of $\Phi_{x}$ will be denoted by $\mathcal{C}_{x}$, and called the variety of minimal rational tangents. The closure of the union of $\mathcal{C}_{x}$ 's over generic points of $X$ forms a subvariety $\mathcal{C} \subset \mathrm{P} T(X)$, called the bundle of varieties of minimal rational tangetns. $\mathcal{C}$ is irreducible, otherwise the collection 
of members of $\mathcal{K}$ tangent to a component of $\mathcal{C}$ will violate the irreducibility of $\mathcal{K}$. From Mori's bend-and-break trick the following is well-known ([Ko, IV.2.9]).

$\left({ }^{*}\right)$ For a generic $\mathcal{K}$-curve $C$, the normalization $\nu: \mathbf{P}_{1} \rightarrow C$ is an immersion and $\nu^{*} T(X)=$ $\mathcal{O}(2) \oplus \mathcal{O}(1)^{p} \oplus \mathcal{O}^{n-1-p}$ for some nonnegative integer $p$ which is equal to the dimension of $\mathcal{K}_{x}$ and $\mathcal{C}_{x}$ for a generic $x \in X$. It follows that for any positive-dimensional family of rational curves belonging to $\mathcal{K}_{x}$, the members of this family do not share a common point other than $x$.

We recall three lemmas about abelian varieties. Recall that when $S \subset A$ is a connected subvariety (not necessarily irreducible) of the abelian variety $A$, the smallest abelian subvariety of $A$ containing all the differences $s-s^{\prime}$, where $s, s^{\prime} \in S$, is called the toroidal hull of $S$, and denoted by $\langle S\rangle$. Note that for any $s, s^{\prime} \in S, S$ is contained in $s+\langle S\rangle=s^{\prime}+\langle S\rangle$. For the proof of the following lemma, see [Za].

Lemma 1 ([Za], p.28, Lemma 3.2) Let $S \subset A=\mathrm{C}^{n} / \Lambda$ be a connected subvariety whose tangent spaces to smooth points are contained in a vector subspace $\mathbf{C}^{m} \subset \mathbf{C}^{n}$. Then the toroidal hull of $S$ has dimension $\leq m$.

The next lemma is a simple consequence of the fact that we cannot have a positive-dimensional family of abelian subvarieties.

Lemma 2 Given any irreducible family of subvarieties of an abelian variety, the toroidal hulls of generic members are identical.

A direct consequence of these two lemmas is

Lemma 3 Let $C_{t} \subset A$ be a p-dimensional irreducible family of curves on an abelian variety passing through a common point $a \in A$. If the union of $C_{t}$ 's covers $a(p+1)$-dimensional constructible subset in $A$ and the subspace of $H^{0}\left(C_{t}, T^{*}(A)\right)$ consisting of elements annihilating tangent vectors to $C_{t}$ has dimension $\geq n-1-p$ for a generic member $C_{t}$, then the closure of the union of these curves is a translate of a $(p+1)$-dimensional abelian subvariety which is the toroidal hull of a generic $C_{t}$.

Lemma 3 shows that the varieties of minimal rational tangents on our $X$ are union of linear subspaces:

Proposition 2 Let $f: A \rightarrow X$ be a finite morphism from an abelian variety onto a uniruled projective manifold. Let $\mathcal{K}$ be as above and let $\mathcal{C}_{x}$ be the variety of minimal rational tangents at a generic point $x \in X$, which has dimension $p$. Then there exist finitely many distinct $(p+1)$ dimensional abelian subvarieties $A_{1}, \ldots, A_{m}$ of $A$ with the following property. Let $\mathcal{A}^{i} \subset T(A)$ be the distribution defined by the translates of $A_{i}$. Then for each generic $x \in X$,

$$
\mathcal{C}_{x}=d f_{a_{j}}\left(\mathbf{P} \mathcal{A}^{1} \cup \cdots \cup \mathbf{P} \mathcal{A}^{m}\right), \quad 1 \leq j \leq d
$$




$$
=d f_{a_{1}}\left(\mathbf{P} \mathcal{A}^{i}\right) \cup \cdots \cup d f_{a_{d}}\left(\mathbf{P} \mathcal{A}^{i}\right), \quad 1 \leq i \leq m
$$

where $f^{-1}(x)=\left\{a_{1}, \ldots, a_{d}\right\}$. In particular, $\mathcal{C}_{x}$ is the union of $m$ linear subspaces of dimension $p$, defining a multi-valued distribution on a Zariski dense open subset of $X$. A local integral submanifold of these distributions at $x \in X$ is given by the image of a translate of $A_{i}$. This local integral submanifold contains an open subset of the variety obtained by the union of members of an irreducible component of $\mathcal{K}_{x}$.

Proof. Choose a generic point $a \in A$ outside the ramification locus of $f$. Let

$$
\mathcal{C}_{x}=\mathcal{C}_{x, 1} \cup \cdots \cup \mathcal{C}_{x, m}
$$

be the decomposition into irreducible components of the variety of minimal rational tangents at $x=f(a)$. Each component $\mathcal{C}_{x, i}$ is the strict image of an irreducible component $\mathcal{K}_{x, i}$ of $\mathcal{K}_{x}$ under $\Phi_{x}: \mathcal{K}_{x} \rightarrow \mathrm{P} T_{x}(X)$. For a rational curve $C_{i} \subset X$ corresponding to a generic point of $\mathcal{K}_{x, i}$, let $C_{i}^{\prime} \subset A$ be an irreducible component of $f^{-1}\left(C_{i}\right)$ through $a$. Note that elements of $H^{0}\left(C_{i}, T^{*}(X)\right)$ annihilates the tangent vectors to $C_{i}$ and $h^{0}\left(C_{i}, T^{*}(X)\right)=n-1-p$ from $\left(^{*}\right)$. The pull-back of elements of $H^{0}\left(C_{i}, T^{*}(X)\right)$ to $H^{0}\left(C_{i}^{\prime}, T^{*}(A)\right)$ gives a subspace of dimension $\geq n-1-p$, annihilating tangent vectors of $C_{i}^{\prime}$. By Lemma 3, the closure of the union of all such choices of $C_{i}^{\prime}$ is $a+<C_{i}^{\prime}>$. It follows that $d f_{a}^{-1}\left(\mathcal{C}_{x, i}\right)=\mathbf{P} T_{a}\left(a+<C_{i}^{\prime}>\right)$. Putting $A_{i}=<C_{i}^{\prime}>$, we get the first equality of the proposition. As for the second equality, the inclusion

$$
d f_{a_{1}}\left(\mathbf{P} \mathcal{A}^{i}\right) \cup \cdots \cup d f_{a_{d}}\left(\mathbf{P} \mathcal{A}^{i}\right) \subset \mathcal{C}_{x}
$$

follows from the first equality. Suppose this inclusion is not an equality for generic $x$. Then the closure of the union of $d f_{a}\left(\mathrm{P} \mathcal{A}^{i}\right)$ for generic $a \in A$ form a subvariety of the bundle of varieties of minimal rational tangents $\mathcal{C}$, which has the same dimension as $\mathcal{C}$, contradicting the irreducibility of $\mathcal{C}$. The statement about the integral submanifold of the distribution follows from the way $A_{i}$ is defined from Lemma 3.

We have the following regularity of the integral subvarieties of this multi-valued distribution.

Proposition 3 Let $a \in A$ be a generic point and $A_{i}$ be as in Proposition 2. Let $Z=f\left(a+A_{i}\right)$ be the image of the translate of $A_{i}$. Then the normalization map $h: \hat{Z} \rightarrow Z$ is an immersion outside a set of codimension $\geq 2$ on $Z$.

Proof. Let $B \subset X$ be the branch divisor of $f: A \rightarrow X$. Certainly $Z$ is smooth outside $B$. Let $D \subset \hat{Z}$ be a component of $h^{-1}(B \cap Z)$. We want to show that $h$ is unramified at a generic point $z$ of $D$.

From Proposition 2, $Z$ is the closure of the union of members of an irreducible component $\mathcal{K}_{x, i}$ of $\mathcal{K}_{x}$ for a generic $x \in Z$. We know that $\mathcal{K}_{x, i}$ for a generic $x$ is a complete family all 
members of which are irreducible. Let $\hat{\mathcal{K}}_{x, i}$ be the family obtained by pulling back $\mathcal{K}_{x, i}$ by $h$. $\hat{\mathcal{K}}_{x, i}$ is a complete family consisting of irreducible rational curves on $\hat{Z}$. A generic member $C$ of $\hat{\mathcal{K}}_{x, i}$ lies on the smooth part of $\hat{Z}$, otherwise we have a 1-dimensional subfamily of curves passing through two distinct points, $h^{-1}(x)$ and a singular point of $\hat{Z}$, a contradiction to $\left.{ }^{*}\right)$. Thus we can think of the tangent bundle $T(\hat{Z})$ in a neighborhood of a generic member $C$. Since $\hat{\mathcal{K}}_{x, i}$ gives deformations of $C$ fixing the point $h^{-1}(x)$ covering the whole $\hat{Z}$, the tangent bundle $T(\hat{Z})$ restricted to a generic member $C$ must be an ample vector bundle ([Ko, II.3.10.1]). Since $C$ is of minimal degree among rational curves on $Z$ through $x$, we have ([Ko, IV.2.9])

$$
\left.T(\hat{Z})\right|_{C}=\mathcal{O}(2) \oplus \mathcal{O}(1)^{p}
$$

For any point $z$ of $D$, we can find a member of $\hat{\mathcal{K}}_{x, i}$ which passes through $z$, because members of $\hat{\mathcal{K}}_{x, i}$ cover $Z$. By the irreducibility of the family, all members of $\hat{\mathcal{K}}_{x, i}$ intersect $D$. It follows that for a generic point $z$ of $D$, we can find a generic member $C$ of $\hat{\mathcal{K}}_{x, i}$ passing through $z$. From the splitting type of $\left.T(\hat{Z})\right|_{C}$ we have $p$-dimensional deformations $C_{s}$ of $C$ passing through $z$. The tangent vectors to these deformations cover a $p$-dimensional open subset in $\mathbf{P} T_{z}(\hat{Z})$.

Suppose $h$ is ramified at $z$. Then $h\left(C_{s}\right)$ is a $p$-dimensional family of deformations of $h(C)$ whose tangent vectors at $h(z)$ can span only $(p-1)$-dimensional subset in $\mathbf{P} T_{h(z)}(X)$. This means that $h(C)$ has a deformation fixing the point $h(z)$ to second order and its Kodaira-Spencer class gives a section of the normal bundle of $h(C)$ vanishing to second order, a contradiction to the splitting type of $T(X)$ on $h(C)$ in $\left(^{*}\right)$.

\section{When the varieties of minimal rational tangents are nondegenerate}

In this section, we will show that if $\mathcal{C}_{x}$ is nondegenerate in $\mathrm{PT}_{x}(X)$ then $X \cong \mathbf{P}_{n}$.

Proposition 4 In the situation of Proposition 2, suppose $\mathcal{C}_{x}$ is nondegenerate in $\mathbf{P} T_{x}(X)$ for a generic $x \in X$, namely, it is not contained in any hyperplane of $\mathbf{P} T_{x}(X)$. Then $m=1$ and $p=n-1$.

Proof. First we claim that $X$ is rationally connected. Otherwise, we have the maximal rational fibration of $X$ over a positive-dimensional variety such that all rational curves through a very general point of $X$ are contained in the fiber through the point ([Ko, IV.5.2]). This implies that $\mathcal{C}_{x}$ should be contained in the tangent space of the fiber through $x$, a contradiction to the nondegeneracy assumption. Since a rationally connected manifold is simply connected, we will prove $m=1$ by constructing an unramified covering of degree $m$ over $X-E$ for some subvariety $E$ of codimension at least 2 . 
From Proposition 3, in an analytic neighborhood $U$ of a generic point $x$ of the branch divisor $B$

$$
U \cap\left(f\left(a+A_{1}\right) \cup \cdots \cup f\left(a+A_{m}\right)\right)=Z_{1} \cup \cdots \cup Z_{l}
$$

for some $l \geq m$, where $a$ is any point of $f^{-1}(x)$ contained in the ramification divisor $R$ and $Z_{j}, 1 \leq j \leq l$, are submanifolds of dimension $p+1$. We want to show that $T_{x}\left(Z_{1}\right) \cup \cdots \cup T_{x}\left(Z_{l}\right)$ has at least $m$ distinct components in $T_{x}(X)$.

Among the $m$ distinct abelian subvarieties $A_{1}, \ldots, A_{m}$, we may assume that $a+A_{1}, \ldots, a+A_{r}$ are contained in $R$ while $a+A_{r+1}, \ldots, a+A_{m}$ are not contained in $R$, for some $0 \leq r \leq m$.

From the genericity of $x \in B$ and $a \in R$, we may assume that $\left.f\right|_{R}$ is unramified at $a$ and the kernel of $d f_{a}$ is a 1-dimensional complement to $T_{a}(R)$ in $T_{a}(A)$. The tangent spaces $T_{a}\left(a+A_{1}\right), \ldots, T_{a}\left(a+A_{r}\right)$ lie in $T_{a}(R)$. We claim that each of $T_{a}\left(a+A_{r+1}\right), \ldots, T_{a}\left(a+A_{m}\right)$ contains the kernel of $d f_{a}$, i.e., $\left.f\right|_{A_{j}+a}$ is ramified at $a$ for all $r+1 \leq j \leq m$. As in the proof of Proposition 3 , there exists a $\mathcal{K}$-curve $C$ on $f\left(a+A_{j}\right)$ which passes through $x$ and is transversal to $B$ at $x$. Let $C^{\prime}$ be an irreducible component of $f^{-1}(C)$ lying on $a+A_{j}$. If $C^{\prime}$ is not tangent to the kernel of $d f_{a}$, then the tangent of $f\left(C^{\prime}\right)$ would be contained in the image of $d f$. This means that $C$ is tangent to $B$, contradictory to our choice of $C$. It follows that the kernel of $d f$ at $a$ is contained in $T_{a}\left(a+A_{j}\right)$.

Thus $T_{a}(R) \cap T_{a}\left(a+A_{j}\right), r+1 \leq j \leq m$ constitute a set of $m-r$ distinct subspaces of dimension $p$ in $T_{a}(R)$. Since $f\left(a+A_{j}\right)$ is immersed at $x$ and $d f_{a}$ sends $T_{a}(R)$ isomorphically to $T_{x}(B)$, we see that $T_{x}\left(f\left(a+A_{j}\right)\right), r+1 \leq j \leq m$ constitute a set of at least $m-r$ distinct subspaces of dimension $p+1$ in $T_{x}(X)$, which are not contained in $T_{x}(B)$. On the other hand, $T_{x}\left(f\left(a+A_{i}\right)\right), 1 \leq i \leq r$ constitute a set of $r$ distinct subspaces of dimension $p+1$ in $T_{x}(B)$. It follows that $T_{x}\left(Z_{1}\right) \cup \cdots \cup T_{x}\left(Z_{l}\right)$ has at least $m$ distinct components.

Let $\operatorname{Gr} T(X)$ be the Grassmann bundle of $(p+1)$-dimensional subspaces of $T(X)$. For a generic $y \in X, \mathcal{C}_{y} \subset \mathbf{P} T_{y}(X)$ consists of $m$ linear subspaces of dimension $p$, defining $m$ distinct points in $\operatorname{Gr} T_{y}(X)$. We denote this set of $m$ distinct points by $\mathcal{S}_{y}$. The closure of the union of the $\mathcal{S}_{y}$ 's gives a subvariety $\mathcal{S} \subset \operatorname{Gr} T(X) . \mathcal{S}$ is irreducible because $\mathcal{C}$ is irreducible. The natural projection $\mathcal{S} \rightarrow X$ is generically $m$-to- 1 . From the above discussion, $\mathcal{S}$ has at least $m$ distinct points over $x \in B$. Thus it is $m$-to-1 over $x$, too. Applying the above argument to generic points of all components of the branch divisor of $f$, we see that $\mathcal{S} \rightarrow X$ is $m$-to-1 outside a set of codimension $\geq 2$ in $X$. Thus $m=1$ from the simple connectedness of $X$. We conclude that $\mathcal{C}_{x}=\mathbf{P} T_{x}(X)$ and $p=n-1$ from the nondegeneracy of $\mathcal{C}_{x}$.

Proposition 5 Under the assumption of Proposition $4, X$ is isomorphic to $\mathbf{P}_{n}$.

Proof. From Proposition $4, X$ is a uniruled projective manifold with a minimal rational 
component $\mathcal{K}$ having $p=n-1$. By [Mk] (2.4), if $X$ is different from $\mathbf{P}_{n}$, then there exists a hypersurface $\mathcal{H}_{x} \subset \mathcal{K}_{x}$ for a generic point $x \in X$, so that a generic member of $\mathcal{H}_{x}$ is a rational curve $C$ for which the pull-back of $T(X)$ to its normalization splits as $\mathcal{O}(2)^{2} \oplus \mathcal{O}(1)^{n-3} \oplus \mathcal{O}$. Let $Z_{x}^{\prime}$ be the hypersurface in $X$ covered by the members of $\mathcal{H}_{x}$. Applying Lemma 3 to the inverse images of members of $\mathcal{H}_{x}$ under $f$ as in the proof of Proposition 2, we see that there exists an abelian hypersurface $H \subset A$ so that the $Z_{x}^{\prime}$ 's are just images of translates of $H$. By the genericity of $x$, we can assume that $Z_{x}^{\prime}$ is smooth at $x$. Since $Z_{x}^{\prime}$ is covered by the members of $\mathcal{H}_{x}, T_{x}\left(Z_{x}^{\prime}\right)$ corresponds to the positive part $\mathcal{O}(2)^{2} \oplus \mathcal{O}(1)^{n-3}$ of the splitting of $T(X)$ on the normalization of $C$. In particular, elements of $H^{0}\left(C, T^{*}(X)\right)$ annihilate $T_{x}\left(Z_{x}^{\prime}\right)$.

We may replace $A$ by an unramified finite cover and assume that $A=E \times H$ for some elliptic curve $E$. Let $C_{t}, t \in \Delta=\{t \in \mathbf{C},|t|<1\}$ be a family of curves belonging to $\mathcal{K}_{x}$ so that $C_{t}$ is generic in $\mathcal{K}_{x}$ for $t \neq 0$ while $C_{0}$ is generic in $\mathcal{H}_{x}$. For $a \in f^{-1}(x)$ let $C_{t}^{\prime}$ be a component of $f^{-1}\left(C_{t}\right)$ through $a$. Then the toroidal hull of $C_{t}^{\prime}, t \neq 0$ is $A$, while the toroidal hull of $C_{0}^{\prime}$ is $H$. Considering small values of $t$, we may assume that $C_{t}^{\prime}$ form a flat family for $t \neq 0$ converging to $C_{0}^{\prime} \cup C^{1} \cup \cdots \cup C^{r}$ for some irreducible curves $C^{j}, 1 \leq j \leq r$ in $A$. Clearly, $C_{0}^{\prime} \cup C^{1} \cup \cdots \cup C^{r}$ is a connected curve and $f\left(C^{j}\right)=C_{0}$ for all $j$. Since $C_{t}^{\prime}, t \neq 0$ surjects to $E$ under the natural projection $E \times H \rightarrow E$, one of $C^{j}$ 's must surject to $E$.

Let $\omega \in H^{0}\left(C_{0}, T^{*}(X)\right)$ be a nonzero element. Pulling back by $\left.f^{*}\right|_{C_{0}^{\prime}}: f^{*}\left(\left.T(X)\right|_{C_{0}}\right) \rightarrow$ $\left.T^{*}(A)\right|_{C_{0}^{\prime}}$, we get a non-zero element $\left(\left.f^{*}\right|_{C_{0}^{\prime}}\right) \omega$ in $H^{0}\left(C_{0}^{\prime}, T^{*}(A)\right)$, which annihilates $T(H)$ because $\omega$ annihilates $T\left(Z_{x}^{\prime}\right)$. From $H^{0}\left(C_{0}^{\prime}, T^{*}(A)\right)=H^{0}\left(A, T^{*}(A)\right),\left(\left.f^{*}\right|_{C_{0}^{\prime}}\right) \omega$ must be the restriction of a parallel 1-form $\Omega \in H^{0}\left(A, T^{*}(X)\right)$ annihilating $T(H)$. From the connectedness of $C_{0}^{\prime} \cup C^{1} \cup \cdots \cup$ $C^{r}$, there exists some $C^{j}$, say $C^{1}$, which intersects $C_{0}^{\prime}$ at some point $b$. Then $\left(\left.f^{*}\right|_{C^{1}}\right) \omega$ is again the restriction of a parallel 1-form $\Omega_{1} \in H^{0}\left(A, T^{*}(A)\right)$. Since $\Omega$ and $\Omega_{1}$ agrees at $b, \Omega_{1}=\Omega$. By the same argument applied to each component of the connected curve $C_{0}^{\prime} \cup C^{1} \cup \cdots \cup C^{r},\left(\left.f^{*}\right|_{C^{j}}\right) \omega$ must be the restriction of $\Omega$ for all $j$. Since $f\left(C^{j}\right)=C_{0},\left(\left.f^{*}\right|_{C^{j}}\right) \omega$ annihilates the tangent vectors to $C^{j}$. This implies that the toroidal hull of $C^{j}$ lies in $H$ for all $j$ and $C^{j}$ cannot surject to $E$ for any $j$, a contradiction.

\section{Structure of the distribution defined by the linear span of varieties of minimal rational tangents}

When the varieties of minimal rational tangents are degenerate, we can use their linear span to define a distribution on $X$. We will show that this distribution defines the structure of a bundle of projective spaces as stated in the Main Theorem. We will say that a meromorphic distribution is integrable if it is integrable over the open set where it defines a genuine holomorphic distribution. 
Proposition 6 In the situation of Proposition 2, let $W_{x} \subset T_{x}(X)$ be the linear span of $\mathcal{C}_{x}$ for a generic $x \in X$. Let $W$ be the meromorphic distribution on $X$ defined by $W_{x}$ at generic $x \in X$. There exists an abelian subvariety $A^{\prime} \subset A$ so that the images of translates of $A^{\prime}$ are integral subvarieties of the distribution $W$.

Proof. $d f^{-1} W$ corresponds to the distribution $\mathcal{W}$ on $A$ defined by the translates of the linear span of $A_{1} \cup \cdots \cup A_{m}$. Since $\mathcal{W}$ is integrable, $W$ is integrable because $f$ is unramified at generic point. By [HM1] Proposition 11, the closure of each leaf of $W$ is a subvariety in $X$. The inverse images of these subvarieties in $A$ are the integral subvarieties of $\mathcal{W}$ and must be the translates of an abelian subvariety $A^{\prime} \subset A$, where $A^{\prime}$ is the toroidal hull of $A_{1} \cup \cdots \cup A_{m}$. Thus integral subvarieties of $W$ are just images of the translates of $A^{\prime}$.

Proposition 7 In the situation of Proposition 6, there exists a fibration $\psi: X \rightarrow Y$ over a normal variety $Y$ such that the underlying reduced variety of each fiber of $\psi$ is the image of a translate of the abelian subvariety $A^{\prime} \subset A$ under $f$. Furthermore, let $\mathcal{K}^{y}$ be the subscheme of $\mathcal{K}$ corresponding to curves contained in the fiber $X_{y}=\psi^{-1}(y)$ over a generic point $y \in Y$. Then there exists a unique component $\mathcal{K}^{\prime}$ of $\mathcal{K}^{y}$ whose members cover $X_{y}$. In particular, $\mathcal{K}^{\prime}$ is a minimal rational component for $X_{y}$ and its variety of minimal rational tangent at a generic point $x \in X_{y}$ coincides with $\mathcal{C}_{x}$.

Proof. Consider an irreducible subscheme $Y$ of the Chow scheme of $X$ whose generic points parametrizes the integral subvarieties of the distribution $W$ in Proposition 6. Let $\phi: \mathcal{F} \rightarrow$ $X, \psi: \mathcal{F} \rightarrow Y$ be the universal family morphisms associated to $Y$ ([Ko, I.3]). By taking reduced structures of the schemes involved and then taking normalization, we may assume that $\mathcal{F}$ and $Y$ are normal. Since $f$ is finite, there exist only a finite number of subvarieties through each point of $X$, which are the images of the translates of $A^{\prime}$. It follows that $\phi$ is a finite morphism. On the other hand, parametrizing the leaves of a foliation, $\phi$ must be birational. Thus $\phi$ is an isomorphism and we get $\psi: X \rightarrow Y$. The underlying reduced varieties of the fibers of $\psi$ are set-theoretic images of translates of $A^{\prime}$.

From the definition of $W$, all members of $\mathcal{K}$ are contained in the fibers of $\psi$. Choose a component $\mathcal{K}^{\prime}$ of $\mathcal{K}^{y}$ whose members cover $X_{y}$. For the uniqueness of $\mathcal{K}^{\prime}$, it suffices to show that the variety of minimal rational tangents $\mathcal{C}_{x}^{\prime}$ associated to $\mathcal{K}^{\prime}$ coincides with $\mathcal{C}_{x}$. By Proposition $2, \mathcal{C}_{x}=d f_{a_{1}}\left(\mathbf{P} \mathcal{A}^{i}\right) \cup \cdots \cup d f_{a_{d}}\left(\mathbf{P} \mathcal{A}^{i}\right)$ where $f^{-1}(x)=\left\{a_{1}, \ldots, a_{d}\right\}$, for the toroidal hull $A_{i}$ of any component of the inverse image of a generic curve in $\mathcal{K}$. By the genericity of $y$, this holds for the toroidal hull $A_{i}$ of any component of the inverse image of a generic curve in $\mathcal{K}^{\prime}$. On the other hand, applying Proposition 2 to $f: a_{1}+A^{\prime} \rightarrow X_{y}$ and $\mathcal{K}^{\prime}$, we see that $\mathcal{C}_{x}^{\prime}=d f_{a_{1}}\left(\mathbf{P} \mathcal{A}^{i}\right) \cup \cdots \cup d f_{a_{d}}\left(\mathbf{P} \mathcal{A}^{i}\right)$, which is exactly $\mathcal{C}_{x}$.

For the minimal rational component $\mathcal{K}^{\prime}$ for $X_{y}$ for generic $y \in Y$ in the above proposition, 
$\mathcal{C}_{x} \subset \mathbf{P} T_{x}\left(X_{y}\right)$ is linearly nondegenerate at generic $x \in X_{y}$. From Proposition 5 , we see that a generic fiber of $\psi: X \rightarrow Y$ is $\mathbf{P}_{p+1}$. This proves Theorem 1. Now by the next Proposition, we can see that $Y$ is smooth and $\psi$ is a $\mathbf{P}_{p+1}$-bundle (using, e.g., [GR, p.212]).

Proposition 8 Let $X$ be a projective algebraic manifold and $\psi: X \rightarrow Y$ be a surjective morphism onto a normal variety. Assume that the underlying reduced variety of each fiber of $\psi$ is irreducible of dimension $k$. If a generic fiber is isomorphic to $\mathbf{P}_{k}$, then each fiber is isomorphic to $\mathbf{P}_{k}$ and $Y$ is smooth.

Proof. Let $o \in Y$ be any point and $V$ be the underlying reduced subvariety of $X_{o}$. At a generic point $x \in V$, choose a local submanifold $S \subset X$ of codimension $k$ transversal to $V$ at $x$. Then $\left.\psi\right|_{S}: S \rightarrow Y$ is a finite holomorphic map. If it is 1-to-1, it is biholomorphic over its image from the normality of $Y$. This implies that $o \in Y$ is a smooth point and $X_{o}$ is Cohen-Macaulay. Moreover considering the intersection number $S \cdot X_{y}, X_{o}$ is generically reduced. It follows that $X_{o}$ is a reduced variety. On the other hand if $\left.\psi\right|_{S}$ is not 1-to-1 for any choice of $S$, then $X_{o}$ cannot be generically reduced and it is a multiple fiber of $\psi$. We will prove Proposition 8 in three steps.

Step 1 Generically reduced fiber over a smooth point

Suppose a fiber $X_{o}$ at a smooth point $o \in Y$ is generically reduced. Then $Y$ is smooth at $o$ and $\psi$ is flat, because morphisms between smooth varieties having equi-dimensional fibers are flat. Moreover $X_{o}$ is a reduced Cohen-Macaulay variety. From the reducedness of $X_{o}, \psi$ is a smooth morphism in a neighborhood of a generic point of $X_{o}$. Thus there exists an open neighborhood $U \subset Y$ of $o$ and an open subset $U^{\prime} \subset X$ in classical topology so that $U^{\prime} \cong U \times \Delta^{k}$ where $\Delta^{k}$ is the polydisc in $\mathbf{C}^{k}$ and $\psi$ corresponds to the projection $U \times \Delta^{k} \rightarrow U$. We can find $k$ disjoint sections $\sigma_{i}: U \rightarrow U^{\prime}, 1 \leq i \leq k$, of $\psi$ such that $\sigma_{1}(y), \ldots, \sigma_{k}(y)$ are in general position in $X_{y} \cong \mathbf{P}_{k}$ for each $y \in U-\{o\}$. The linear span of the $\sigma_{i}(y)$ 's in $X_{y}, y \neq o$, determines a unique hyperplane $L_{y}$ on $X_{y} \cong \mathbf{P}_{k}$. Taking the closure of the union of $L_{y}$ 's, we get a hypersurface $L$ in $\psi^{-1}(U)$ whose restriction to $X_{y}, y \neq o$, is a hyperplane. $L$ defines a line bundle on $X_{o}$ with $L^{k} \cdot X_{o}=1$ and $\operatorname{dim}\left(H^{0}\left(X_{o}, L\right)\right) \geq k+1$ from the flatness of $\psi$. Thus $X_{o} \cong \mathbf{P}_{k}$ by [Fu, I.1.1].

Step 2 Multiple fibers along a hypersurface of $Y$

Suppose $\psi$ has multiple fibers along a hypersurface $D$ in $Y$ with generic multiplicity $r>1$. Let $o$ be a generic point of $D$ which is a smooth point of $Y$. Let $V$ be the underlying variety of $X_{o}$. At a generic point $x \in V$, choose a local submanifold $S \subset X$ of codimension $k$ transversal to $V$ at $x$. Then $\left.\psi\right|_{S}$ is ramified over $D$. We can choose a coordinate system $\left(s_{1}, \ldots, s_{n-k}\right)$ on $S$ and $\left(y_{1}, \ldots, y_{n-k}\right)$ on a smooth open neighborhood $U=\psi(S) \subset Y$ of $o$, so that $\left.\psi\right|_{s}$ is given by $y_{1}=s_{1}^{r}, y_{2}=s_{2}, \ldots, y_{n-k}=s_{n-k}$. There exists a proper subvariety $W \subset V$ such that at any point of $V-W$, there exists a germ $g$ of holomorphic functions on $X$ satisfying $g^{r}=\psi^{*} y_{1}$. 
Let $\tilde{\psi}: \mathcal{H}^{\prime} \rightarrow S$ be the pull-back of $\left(\psi^{-1}(U)-W\right) \rightarrow U$ by $\left.\psi\right|_{S}$, defined by $\mathcal{H}^{\prime}:=\{(s, z) \in$ $\left.S \times\left(\psi^{-1}(U)-W\right), \psi(s)=\psi(z)\right\} . \mathcal{H}^{\prime}$ is locally defined by the equations

$$
s_{1}^{r}-\psi^{*} y_{1}=0, s_{2}-\psi^{*} y_{2}=0, \ldots, s_{n-k}-\psi^{*} y_{n-k}=0 .
$$

Since we are excluding $W$,

$$
s_{1}^{r}-\psi^{*} y_{1}=s_{1}^{r}-g^{r}=\prod_{\zeta^{r}=1}\left(s_{1}-\zeta g\right) .
$$

So the normalization $\mathcal{H}$ of $\mathcal{H}^{\prime}$ is smooth and the natural morphism $\rho: \mathcal{H} \rightarrow\left(\psi^{-1}(U)-W\right)$ is an unramified $r$-to- 1 cover of $\psi^{-1}(U)-W$.

Since $W$ is of codimension $\geq 2$ in $\psi^{-1}(U), \pi_{1}\left(\psi^{-1}(U)\right)=\pi_{1}\left(\psi^{-1}(U)-W\right)$. So there exists a complex manifold $\overline{\mathcal{H}}$ and an unramified $r$-to-1 covering $\overline{\mathcal{H}} \rightarrow \psi^{-1}(U)$ extending $\rho$. The morphism $\tilde{\psi}: \mathcal{H} \rightarrow S$ extends to $\bar{\psi}: \overline{\mathcal{H}} \rightarrow S$ by Hartogs. By construction, $\bar{\psi}$ has no multiple fiber and generic fiber is $\mathbf{P}_{k}$. Thus $\bar{\psi}$ is a $\mathbf{P}_{k}$-bundle over $S$ by Step 1. But the fiber of $\bar{\psi}$ over $x \in S$ must be a finite unramified cover of $V$ while $\mathbf{P}_{k}$ cannot be a non-trivial unramified cover of a variety, a contradiction.

Step 3 From Step 1 and Step 2, we may assume that $\psi: X \rightarrow Y$ is a $\mathbf{P}_{k}$-bundle outside a subvariety $E \subset Y$ of codimension $\geq 2$.

Let $o \in E$ and $V$ be the underlying subvariety of $X_{o}$. At a generic point $x \in V$, we choose a local submanifold $S \subset X$ of codimension $k$ transversal to $V$ at $x$. If the morphism $\left.\psi\right|_{S}: S \rightarrow Y$ is 1-to- 1 , then $X_{o}$ is generically reduced and $o \in Y$ is smooth. So $X_{o} \cong \mathbf{P}_{k}$ by Step 1 . Let $\left.\psi\right|_{S}$ be $l$-to- 1 for some $l>1$.

$S$ is transversal to the underlying subvariety of any fiber $X_{y}$ for $y$ close to $o$. Thus $S$ is transversal to $X_{y}$ for $y \notin E, y$ close to $o$. So $\left.\psi\right|_{S}: S \rightarrow U=\psi(S)$ is unramified outside $E$.

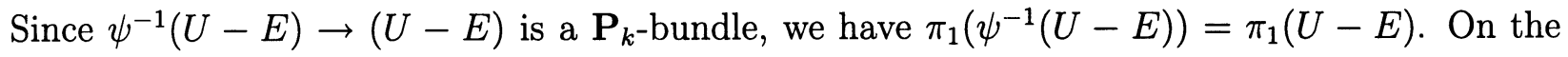
other hand, $\pi_{1}\left(\psi^{-1}(U-E)\right)=\pi_{1}\left(\psi^{-1}(U)\right)$ since $\psi^{-1}(U)$ is smooth and $\psi^{-1}(E)$ is of codimension $\geq 2$ in $\psi^{-1}(U)$. Thus the unramified covering $\left.\psi\right|_{S-\psi^{-1}(E)}:\left(S-\psi^{-1}(E)\right) \rightarrow(U-E)$ induces an unramified covering $\rho: Q \rightarrow \psi^{-1}(U)$ where $Q$ is a complex manifold and $Q^{\prime}:=\rho^{-1}\left(\psi^{-1}(U-E)\right)$ is a $\mathbf{P}_{k}$-bundle over $S-\psi^{-1}(E)$. By Hartogs, we have a natural morphism $\mu: Q \rightarrow S$. The underlying reduced variety of each fiber of $\mu$ is irreducible because $\rho$ is unramified and each fiber of $\psi^{-1}(U)$ is irreducible. Moreover $\mu$ admits a section arising from $\rho^{-1}(S)$. Thus by Step $1, Q$ is a $\mathbf{P}_{k}$-bundle over $S$ while the fiber over $x \in S$ must be an unramified cover of $V$, a contradiction as in Step 2.

By above we have a $\mathbf{P}_{p+1}$-bundle structure $\psi: X \rightarrow Y$ so that each fiber is the image of a translate of an abelian subvariety $A^{\prime} \subset A$ under $f$. To finish the proof of Main Theorem, it remains to show that $Y$ is the image of a finite morphism from an abelian variety. By taking 
a finite unramified covering, we may write $A=A^{\prime} \times A^{\prime \prime}$ for some abelian subvariety $A$ ". Since $f\left(A^{\prime \prime}\right)$ intersects any fiber of $\psi$ at only finitely many points, $\psi \circ f$ gives a finite morphism from an abelian variety $A$ " onto $Y$ and we are done.

\section{References}

[De] Debarre, O.: Images lisses d'une variété abélienne simple. C.R. Acad. Sci. Paris, 309. Série I (1989) 119-122

[Fu] Fujita, T.: Classification theories of polarized varieties. London Math. Soc. Lecture Note Series, vol. 155, Cambridge University Press, 1990

[GR] Grauert, H. and Remmert, R.: Coherent analytic sheaves. Grundlehren der mathematischen Wissenschaften, Band 265, Springer Verlag, 1984

[HM1] Hwang, J.-M. and Mok, N.: Rigidity of irreducible Hermitian symmetric spaces of the compact type under Kähler deformation. Invent. math. 131 (1998) 393-418

[HM2] Hwang, J.-M. and Mok, N.: Holomorphic maps from rational homogeneous spaces of Picard number 1 onto projective manifolds. Invent. math. 136 (1999) 209-231

[Ko] Kollár, J.: Rational curves on algebraic varieties. Ergebnisse der Mathematik und ihrer Grenzgebiete, 3 Folge, Band 32, Springer Verlag, 1996

[La] Lazarsfeld, R. : Some applications of the theory of positive vector bundles. in Complete intersections, Lecture Note in Math. vol. 1092, Springer Verlag, 1984

[Mk] Mok, N.: The uniformization theorem for compact Kähler manifolds of nonnegative holomorphic bisectional curvature. J. Diff. Geom. 27 (1988) 179-214

[MM] Miyaoka, Y. and Mori, S.: A numerical criterion for uniruledness. Ann. Math. 124 (1986) 65-69

[Mo] Mori, S.: Projective manifolds with ample tangent bundles. Ann. Math. 110 (1979) 593-606

[Ue] Ueno, K.: Classification theory of algebraic varieties and compact complex spaces. Lect. Notes in Math. vol. 439, Springer Verlag, 1975

[Za] Zak, F.L.: Tangents and secants of algebraic varieties. Translations of mathematical monographs, vol. 127, American Mathematical Society, 1993

Jun-Muk Hwang

Korea Institute for Advanced Study

207-43 Cheongryangri-dong

Seoul 130-012, Korea

e-mail: jmhwang@ns.kias.re.kr
Ngaiming Mok

Department of Mathematics

The University of Hong Kong

Pokfulam Road, Hong Kong

e-mail: nmok@hkucc.hku.hk 